\title{
Glucagon-Like Peptide-1 Receptor Agonist Treatment Attributes Important to Injection-Experienced Patients with Type 2 Diabetes Mellitus: A Preference Study in Germany and the United Kingdom
}

Lei Qin · Stephanie Chen · Emuella Flood · Alka Shaunik •

Beverly Romero · Marie de la Cruz · Cynthia Alvarez · Susan Grandy

Received: January 3, 2017 / Published online: February 24, 2017

(C) The Author(s) 2017. This article is published with open access at Springerlink.com

\section{ABSTRACT}

Introduction: This study assessed the relative importance of treatment-related attributes in influencing patient preferences for glucagonlike peptide-1 receptor agonists (GLP-1RAs) among injection-experienced type 2 diabetes mellitus (T2DM) patients in Germany and the United Kingdom.

Methods: T2DM patients experienced with injecting once-weekly (QW) exenatide or once-daily (QD) liraglutide completed an online discrete-choice experiment (DCE) survey. Patients chose between hypothetical blinded treatment profiles reflecting attributes of GLP-1RAs. The DCE survey included eight

Enhanced content To view enhanced content for this article go to http://www.medengine.com/Redeem/ 4C87F060093A75A0.

Electronic supplementary material The online version of this article (doi:10.1007/s13300-017-0237-8) contains supplementary material, which is available to authorized users.

L. Qin $(\bowtie) \cdot$ S. Chen · A. Shaunik · S. Grandy AstraZeneca, Gaithersburg, MD, USA e-mail: lei.qin@astrazeneca.com

E. Flood · B. Romero · M. de la Cruz ICON plc, Clinical Outcomes Assessment, Gaithersburg, MD, USA

C. Alvarez

ICON plc, Medical Affairs Statistical Analysis, San Diego, CA, USA attributes: efficacy, side effects, device size, needle size, titration, injection preparation, long-term efficacy/safety, and dosing frequency. Odds ratios (ORs) and 95\% confidence intervals (CIs) were calculated using a conditional logit model indicating the likelihood of choosing a treatment with a given attribute level versus a reference attribute level.

Results: 510 GLP-1RA injection-experienced patients completed the survey; $45.3 \%$ respondents were being treated with exenatide QW and $54.7 \%$ respondents were being treated with liraglutide QD. In terms of GLP-1RA attributes, patients indicated a preference for a treatment with greater efficacy (i.e., a 1.5-point improvement in HbA1c) (OR 2.58; 95\% CI 2.37, 2.80; $p<0.001$ ), fewer side effects (OR 2.67; 95\% CI $2.52,2.82 ; p<0.001)$, once-weekly rather than once-daily administration (OR 2.26; 95\% CI $2.13,2.39 ; p<0.001)$, and the preparation required for a multi-use pen (OR 1.71; 95\% CI $1.55,1.88 ; p<0.001)$. Needle size, device size, and titration were not significant drivers of patient preference.

Conclusions: Among GLP-1RA injection-experienced patients, key drivers of treatment preference for a hypothetical GLP-RA profile were side effects, efficacy, dosing frequency, and required preparation. Understanding patient preferences is important for optimizing treatment decision-making and improving treatment adherence.

Funding: AstraZeneca. 
Keywords: Conjoint; Discrete-choice experiment; Dosing frequency; Exenatide; Glucagon-like peptide-1 receptor agonists; Liraglutide; Treatment preference; Type 2 diabetes mellitus

\section{INTRODUCTION}

The global prevalence of diabetes has been steadily increasing for the last 30 years [1-3]. In 2015, 415 million people were estimated to have diabetes across the globe [4]. By 2040, this number is expected to increase to 642 million [4]. The highest national prevalence rates of diabetes are found in countries in Oceania, followed by the Middle East and North Africa [1]. In these regions, the prevalence of diabetes is five to ten times higher than the lowest prevalence rates found in some Western European countries. Nevertheless, even in Western European countries, diabetes has become increasingly prevalent [1]. Studies have indicated an increase in prevalence from $2.39 \%$ in 2000 to $5.32 \%$ in 2013 in the United Kingdom (UK) [5], from $6.6 \%$ in 2007 to $8.6 \%$ in 2010 in southwestern Germany [6], and from $2.8 \%$ in 1990 to $4.6 \%$ in 2010 in Stockholm, Sweden [7]. In high-income countries, approximately $87-91 \%$ of diabetes patients have type 2 diabetes mellitus (T2DM) [3, 4], so the increases seen in the prevalence of diabetes are likely to be due to the increase in T2DM [1].

A patient-centered approach to the treatment of T2DM is advised [8-10], with the choice of diabetes therapy being based on attributes specific to both patients and the medication. Such an approach balances the benefits of glycemic control against the potential risks (e.g., weight gain, hypoglycemia, cost) of any medication, while also taking into account factors relevant to the patient (e.g., age, health status, preferences, therapeutic goal, and likely adherence) [8-12].

Glucagon-like peptide-1 receptor agonists (GLP-1RAs) are one of the options recommended for the treatment of T2DM [8-10]. GLP-1RAs mimic GLP-1, a gut-derived incretin hormone which increases insulin secretion, inhibits glucagon secretion, increases satiety, and slows gastric emptying [13-15]. These agents reduce fasting and postprandial glucose and glycated hemoglobin A1c (HbA1c) levels but are associated with a low risk of hypoglycemia because they act in a glucose-dependent manner $[15,16]$. Their use has also been associated with bodyweight reduction. The GLP-1RAs differ in their molecular structure and degree of homology to endogenous GLP-1 [17], and therefore some variations in their effects on glycemic control and side effects have been reported $[15,17,18]$. The GLP-1RAs also differ in their frequency of dosing and types of delivery systems.

Given the differences between the GLP-1RAs, it is important to understand patients' preferences for the clinical and nonclinical features of these agents. Such an understanding is important given the high level of non-adherence to T2DM medication [19-24], and the consequent compromise in outcomes $[20,25,26]$. If clinical decisions take into consideration patient preference, this is likely to be reflected in greater adherence to treatment and improved clinical outcomes [9, 27].

Discrete-choice experiments (DCEs) have been used to quantify patients' preferences for the various attributes of diabetes treatments [28-35]. A DCE can provide useful information regarding patients' preferences when they are making very specific medication choices. Previous DCE studies have investigated the attributes of GLP-1RAs, including efficacy (percentage of patients who reach their HbA1c goals) and tolerability (weight gain, nausea, and hypoglycemia), as well as injection-related features such as type of injection device or injection frequency [30, 31, 33]. Further investigation is warranted to gain insight into other attributes (such as device size, preparation required to deliver the medication, requirement for titration, and evidence for long-term efficacy and safety) that may be influencing patients' preferences for one GLP-1RA over another.

The objective of this study was to assess the relative importance of treatment-related attributes in influencing preferences for GLP-1RAs among GLP-1RA injection-experienced patients with T2DM in Germany and the United Kingdom (UK). Two commonly prescribed GLP-1RAs 
[once-weekly (QW) exenatide and once-daily (QD) liraglutide] were chosen to represent hypothetical treatment profiles, each with differing attributes.

\section{METHODS}

\section{Study Design}

This was a two-stage descriptive study that included an initial survey development phase (in both injection-naïve and injection-experienced T2DM patients), followed by a second phase involving a one-time, web-based multi-country DCE survey involving hypothetical treatment profiles, in GLP-1 RA injection-experienced T2DM patients (reported here) and in injection-naïve T2DM patients (reported elsewhere [36]).

The DCE survey was developed in line with best practice guidance $[37,38]$. Using this approach, the key characteristics (attributes) of alternative treatments are identified and a series of levels for each attribute are then selected. Participants are then asked to choose from several hypothetical options, each of which details a series of attributes at different levels. This approach can provide information about the relative importance of the attributes to the patients and their willingness to accept tradeoffs between the different attributes.

The survey was programmed and hosted by Global Perspectives (Berkshire, UK) on a secure server. Eligible participants were identified by a research recruitment agency using multiple methods including reviewing patient databases of those agreeable to contact for research study participation, encouraging referrals from clinicians and patient associations, and advertising in targeted publications. All eligible patients provided online consent through the web link prior to completing the survey. Patients who completed the survey received compensation for their participation.

The study protocol was approved by the Salus Institutional Review Board (Austin, TX, USA). All procedures followed were in accordance with the ethical standards of the responsible committee on human experimentation (institutional and national) and with the Helsinki Declaration of 1964 , as revised in 2013. Informed consent was obtained from all patients before they were included in the study.

\section{Phase One: Survey Development Stage}

A literature review and qualitative research with T2DM patients were conducted to inform attribute selection and survey development. Identification of the key attributes of GLP-1RAs were based on open-ended, one-on-one qualitative interviews conducted with fifty T2DM patients from five countries (Brazil, China, Germany, Japan, and the UK) to identify and examine treatment attributes important to T2DM patients [39].

Eight attributes of GLP-1RAs were chosen for inclusion in a draft DCE survey: common GLP-1RA side effects, dosing frequency, required injection preparation, improvement in $\mathrm{HbA1c}$, requirement for titration, evidence of long-term efficacy/safety [40], injection device size, and needle size. The US Food and Drug Administration-approved labels of two common GLP-1RAs (exenatide QW and liraglutide QD) were reviewed to provide hypothetical base-case treatment profiles $[41,42]$.

One treatment was a profile of a daily injectable approximating-liraglutide QD. The other treatment was a weekly injectable approximating-exenatide QW. Exenatide QW is delivered once-weekly, does not require titration, and uses a thicker, longer needle. Delivery of exenatide was specified as either using a single-use pen device or via an auto-injector. The single-use pen device contains exenatide microspheres and aqueous-based diluent in separate chambers, requiring careful preparation of the medication, while the auto-injector has the exenatide microspheres and lipid-based diluent prefilled in one chamber (see Fig. S1 in the Electronic supplementary material, ESM, for details). Exenatide QW can also be delivered in a single-dose tray (vial and syringe). Liraglutide QD is delivered once-daily via a multiple-use pen, requires careful preparation and titration of the medication (see Fig. S1 in the ESM for details), and uses a thinner, shorter needle. Further details of the attributes and the levels 
assigned to each potential attribute are shown in Table 1.

The attributes and levels were combined into choice sets using a published orthogonal array [43]. An orthogonal fractional factorial design was used to identify the minimum specification of the combinations of attributes and levels that could define the hypothetical treatments [43]. The combinations were then paired using a fold-over design [44]. Each choice question presented two hypothetical treatments (treatment A or B) that reflected attributes associated

Table 1 Attributes and levels included in the discrete-choice experiment survey

\begin{tabular}{|c|c|}
\hline Attribute & Levels \\
\hline Side effects & $\begin{array}{l}\text { Side-effects profile B: } 21 \% \text { chance of nausea, } 13 \% \text { chance of diarrhea, } 11 \% \text { chance of vomiting, } \\
\text { and } 1 \% \text { chance of injection-site nodules (reference) } \\
\text { Side-effects profile A: } 9 \% \text { chance of nausea, } 6 \% \text { chance of diarrhea, } 4 \% \text { chance of vomiting, } \\
\text { and } 10 \% \text { chance of injection-site nodules }{ }^{\mathrm{a}}\end{array}$ \\
\hline Dosing frequency & $\begin{array}{l}\text { Once a day }(\text { reference })^{\mathrm{b}} \\
\text { Once a week }\end{array}$ \\
\hline Preparation required & $\begin{array}{l}\text { Vial and syringe (reference) } \\
\text { Multi-use pen }{ }^{\mathrm{b}} \\
\text { Single-use pen }{ }^{\mathrm{a}} \\
\text { Auto-injector }\end{array}$ \\
\hline Improvement in $\mathrm{HbAlc}$ & $\begin{array}{l}\text { 0.8-point improvement in } \mathrm{HbAlc} \text { (reference) } \\
\text { 1.2-point improvement in } \mathrm{HbAlc} c^{\mathrm{a}, \mathrm{b}} \\
\text { 1.5-point improvement in } \mathrm{HbAlc}\end{array}$ \\
\hline Titration & $\begin{array}{l}\text { Requires titration (reference) } \\
\text { Does not require titration }^{\mathrm{a}}\end{array}$ \\
\hline $\begin{array}{l}\text { Evidence of effectiveness } \\
\text { and safety }\end{array}$ & $\begin{array}{l}1 \text { year }(\text { reference }) \\
2 \text { years }^{\mathrm{b}} \\
6 \text { years }^{\mathrm{a}}\end{array}$ \\
\hline Device size & $\begin{array}{l}\text { Single-use pen }{\text { (reference })^{\mathrm{a}}} \\
\text { Multi-use } \text { pen }^{\mathrm{b}} \\
\text { Auto-injector } \\
\text { Vial and syringe }\end{array}$ \\
\hline Needle size & $\begin{array}{l}\text { Longer and thicker }(\text { reference })^{\mathrm{a}} \\
\text { Shorter and thinner }\end{array}$ \\
\hline
\end{tabular}

HbAlc glycated hemoglobin, $Q D$ once-daily, $Q W$ once-weekly

a Attribute associated with exenatide QW; delivery of exenatide QW is achieved using either a single-use pen or an auto-injecting single-use device containing exenatide microspheres and diluent prefilled into separate chambers

b Attribute associated with liraglutide QD 
with exenatide QW and liraglutide QD. A total of 32 pairs of choice sets were generated, with each respondent being presented with a set of
16 unique paired choice sets [45]. An example of a choice set included in the survey is provided in Fig. 1.
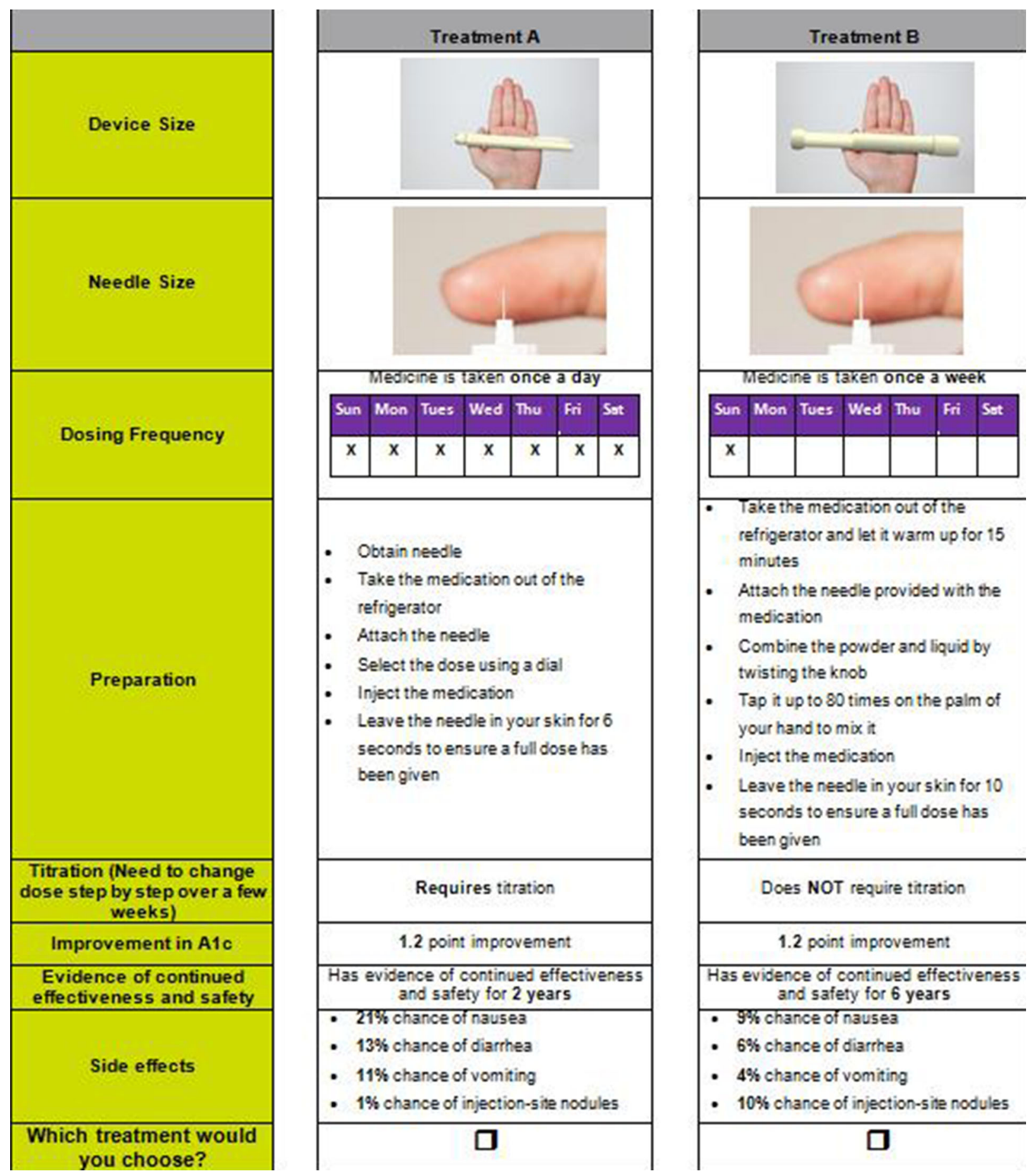

Fig. 1 Example of a discrete-choice experiment question comparing treatment profiles reflecting liraglutide QD and exenatide QW (treatment A vs. treatment B) 
The English-language survey was pilot-tested with respect to wording and comprehension as well as cognitive and overall burden with five T2DM patients in the UK. Revisions were made to improve clarity. The survey was then translated into German and reviewed with respect to wording and comprehension by two independent native German speakers so that it could be used in Germany.

\section{Phase Two: Web-Based DCE Study}

In the second phase of the study, GLP-1RA injection-experienced patients from the UK and Germany completed the one-time, web-based survey. Only patients from the UK and Germany were included, as GLP-1RAs were not readily available in the other countries at the time of the study inception. Interested participants were provided with an internet link to the online web-based DCE survey.

The survey included screening questions and questions relating to past and current medication use, adherence, clinical history, and demographic information. The adherence questions asked about frequency of missing, skipping, or being late in taking their injected GLP-1RA, and what aspect of their current medication made it most difficult or inconvenient to take as prescribed. In addition, participants were asked hypothetical questions about the potential impact of dosing frequency on their adherence.

The second section of the survey included the DCE questions. The DCE began by presenting participants with descriptions of the attributes and the levels included in the survey, followed by the 16 paired comparison questions.

\section{Survey Participants}

In the second phase of the study, the DCE survey was completed by T2DM patients from the UK and Germany who were currently using GLP-1RA injections (exenatide QW or liraglutide QD) for more than 2 months prior to screening (i.e., GLP-1RA injection-experienced). Other eligibility criteria included: age 18 years or older; adequate written and oral fluency in the target language; be willing and able to complete a 30-min online survey about treatment experiences and preferences; have access to the Internet; and be willing to provide informed consent. Patients with type 1 diabetes mellitus, who were pregnant, or who were currently on insulin therapy were excluded from the survey.

\section{Data Analyses}

SAS $^{\circledR}$ statistical software, version 4.3 (SAS Institute Inc., Cary, NC, USA) was used to conduct the analyses. Descriptive statistics [means, standard deviations (SD), ranges, frequencies, and percentages] were reported for the demographic and clinical characteristics data and the self-reported data on adherence to medication. The DCE responses were analyzed using a conditional or mixed logit model, with each attribute and level included as a separate variable in the model. The choice of treatment was the dependent variable and was analyzed conditionally for each choice set (i.e., each treatment choice the respondent had to make). The conditional logit model provided regression coefficients (i.e., utility estimates), which were then presented as odds ratios (ORs) and 95\% confidence intervals (CI). High ORs indicated a strong preference for that attribute level versus a reference attribute level. All analyses were performed for the total sample and separately for each country.

Sensitivity analyses were conducted to examine how much a product's overall preference was improved or worsened by changing certain attributes while holding all other attributes at constant base case levels. For example, the exenatide QW profile was compared to the liraglutide QD profile, assuming better efficacy for liraglutide QD versus exenatide QW. The exenatide QW profile assuming an auto-injector device size and auto-injector preparation was also compared to the liraglutide QD profile. 


\section{RESULTS}

\section{Sample Characteristics}

A total of 510 GLP-1RA injection-experienced patients completed the survey (Table 2). The mean age of the patients in the study was 57.0 years, and $51.4 \%$ were male. The majority was Caucasian, employed, and had some formal education (Table 2). On average, the duration of the T2DM of the participants was 7.2 years.

\section{Current Treatments}

For their clinical care, $45.3 \%$ respondents were receiving treatment with exenatide $\mathrm{QW}$ and $54.7 \%$ respondents were treated with liraglutide QW, using a vial and syringe (10.8\%), disposable single-use pen (40.6\%), or a disposable multiple-use pen (48.6\%). Of the injection-experienced patients, $17.5 \%$ were using one medication only, $56.9 \%$ were using two medications, and $25.7 \%$ were using three or more medications. The majority of participants were also using metformin to treat their diabetes $(64.5 \%)$. The sociodemographic and clinical characteristics were generally similar between patients from the UK and Germany (Table 2).

Overall, $52.2 \%$ of respondents indicated that they never missed a dose of their injectable medication. More of the patients who were treated with liraglutide QD (53.8\%) than exenatide QW (40.7\%) reported missing a dose (Fig. 2). The percentages of the exenatide QW and liraglutide QD recipients who missed a dose at least once a month were $3.9 \%$ and $20.1 \%$, respectively.

The top reasons for patients missing an injection included forgetting (75.1\%), not having the medication with them at the scheduled time (29.8\%), a change in daily routine (29.0\%), not taking the medication out of the refrigerator at the scheduled time (28.6\%), and running out of medication (18.0\%). More than half $(59.4 \%)$ reported taking medication late, with the reasons including forgetting (59.1\%), not taking the medication out of the refrigerator at the scheduled time (37.3\%), experiencing a change in daily routine $(35.3 \%)$, or not having the medication with them at the scheduled time $(30.7 \%)$. When patients were asked about hypothetical injectable T2DM treatments, $72.0 \%$ of respondents $(92.2 \%$ exenatide and $55.2 \%$ liraglutide) stated that a once-weekly dose would be easiest to follow, while $27.1 \%$ of respondents $(6.1 \%$ exenatide and $44.4 \%$ liraglutide recipients) selected a once-daily injection, and $1.0 \%$ of all respondents thought a twice-daily injection would be easiest to follow.

\section{Attributes Influencing Choice of Injectable Medication}

Side effects, efficacy, dosing frequency, and required preparation were the four most important attributes influencing patient preference for an injectable GLP-1RA medication, according to analyses pooled from both countries and from country-specific data from the UK or Germany (Table 3). Across both countries, patients indicated a preference for a treatment associated with fewer side effects (i.e., less nausea, diarrhea, vomiting, or injection-site nodules), a treatment offering greater efficacy (i.e., a 1.5-point improvement in HbA1c), a treatment that was administered once-weekly, and the preparation required for a multi-use pen. German respondents indicated that greater efficacy (i.e., a 1.5-point improvement in HbA1c) was the most valued attribute, followed by side effects, dosing frequency, and required preparation. The UK respondents indicated the level of side effects to be the most important influence on their preference for a treatment, followed by efficacy, preparation required, and dosing frequency (Table 3 ).

In terms of the five device-related attributes, dosing frequency and the preparation required were significant predictors of patient preference according to the pooled and country-specific outcomes. As mentioned above, patients preferred weekly to daily dosing (Table 3 ). Patients indicated a preference for the preparation required for a multi-use pen and an auto-injector to that of a vial and syringe, according to pooled data from both countries. Needle size, device size, and titration were not significant 
Table 2 Self-reported sociodemographic and clinical characteristics of injection-experienced type 2 diabetes mellitus participants

\begin{tabular}{|c|c|c|c|}
\hline Patient characteristic & Total $(N=510)$ & UK $(n=220)$ & Germany $(n=290)$ \\
\hline Mean age (SD), years [range] & $57.0(11.0)[20.0,81.0]$ & $53.0(11.5)[20.0-76.0]$ & $60.0(9.6)[31.0-81.0]$ \\
\hline Male, $n(\%)$ & $262(51.4)$ & $118(53.6)$ & $144(49.7)$ \\
\hline Female, $n(\%)$ & $248(48.6)$ & $102(46.4)$ & $146(50.3)$ \\
\hline \multicolumn{4}{|l|}{ Race/ethnicity, $n$ (\%) } \\
\hline Caucasian & $477(93.5)$ & $207(94.1)$ & $270(93.1)$ \\
\hline Mixed/multiple ethnic groups & $4(0.8)$ & $1(0.5)$ & $3(1.0)$ \\
\hline Asian/Asian British & $17(3.3)$ & $6(2.7)$ & $11(3.8)$ \\
\hline Black/African/Caribbean/Black British & $8(1.6)$ & $5(2.3)$ & $3(1.0)$ \\
\hline Other & $5(1.0)$ & $0(0.0)$ & $4(1.4)$ \\
\hline \multicolumn{4}{|l|}{ Employment status, $n$ (\%) } \\
\hline Employed, full-time & $180(35.3)$ & $81(36.8)$ & $99(34.1)$ \\
\hline Employed, part-time & $66(12.9)$ & $25(11.4)$ & $41(14.1)$ \\
\hline Self-employed & $26(5.1)$ & $13(5.9)$ & $13(4.5)$ \\
\hline Stay-at-home parent/homemaker & $21(4.1)$ & $8(3.6)$ & $13(4.5)$ \\
\hline Unemployed & $23(4.5)$ & $14(6.4)$ & $9(3.1)$ \\
\hline Retired & $159(31.2)$ & $46(20.9)$ & $113(39.0)$ \\
\hline Disabled & $32(6.3)$ & $30(13.6)$ & $2(0.7)$ \\
\hline Other & $3(0.6)$ & $3(1.4)$ & $0(0.0)$ \\
\hline \multicolumn{4}{|l|}{ Education, $n(\%)$} \\
\hline No formal qualifications & $48(9.4)$ & $19(8.6)$ & $29(10.0)$ \\
\hline GCSE/O-level or equivalent & $224(43.9)$ & $66(30.0)$ & $158(54.5)$ \\
\hline A-level or equivalent & $126(24.7)$ & $56(25.5)$ & $70(24.1)$ \\
\hline University degree & $83(16.3)$ & $55(25.0)$ & $28(9.7)$ \\
\hline Postgraduate degree & $29(5.7)$ & $24(10.9)$ & $5(1.7)$ \\
\hline Mean weight, $\mathrm{kg}(\mathrm{SD})$ & $101.7(23.7)$ & $108.0(28.0)$ & $96.9(18.4)$ \\
\hline Mean height, $\mathrm{m}$ (SD) & $1.7(0.1)$ & $1.7(0.1)$ & $1.7(0.1)$ \\
\hline Mean BMI $\left(\mathrm{kg} / \mathrm{m}^{2}\right),(\mathrm{SD})$ & $34.2(7.5)$ & $36.7(9.2)$ & $32.3(5.1)$ \\
\hline Mean duration of T2DM, years (SD) & $7.2(5.9)$ & $9.9(7.1)$ & $5.1(3.6)$ \\
\hline Mean $\mathrm{HbA}_{1 \mathrm{c}}(\mathrm{SD}), \%^{\mathrm{a}}$ & $7.4(1.9)$ & $7.9(2.9)$ & $7.1(0.7)$ \\
\hline \multicolumn{4}{|l|}{ Current GLP-1RA treatment } \\
\hline Exenatide QW, $n(\%)$ & $231(45.3)$ & $84(38.2)$ & $147(50.7)$ \\
\hline
\end{tabular}


Table 2 continued

\begin{tabular}{llll}
\hline Patient characteristic & Total $(\boldsymbol{N}=\mathbf{5 1 0})$ & UK $(\boldsymbol{n}=\mathbf{2 2 0})$ & Germany $(\boldsymbol{n}=\mathbf{2 9 0})$ \\
\hline Liraglutide QD, $n(\%)$ & $279(54.7)$ & $136(61.8)$ & $143(49.3)$ \\
\hline
\end{tabular}

$B M I$ body mass index, GLP-1RA glucagon-like peptide-1 receptor agonist, $H b A 1 c$ hemoglobin Alc, $Q D$ once-daily, $Q W$ once-weekly, $S D$ standard deviation, $T 2 D M$ type 2 diabetes mellitus

a $n=109$ (UK) and 198 (Germany)

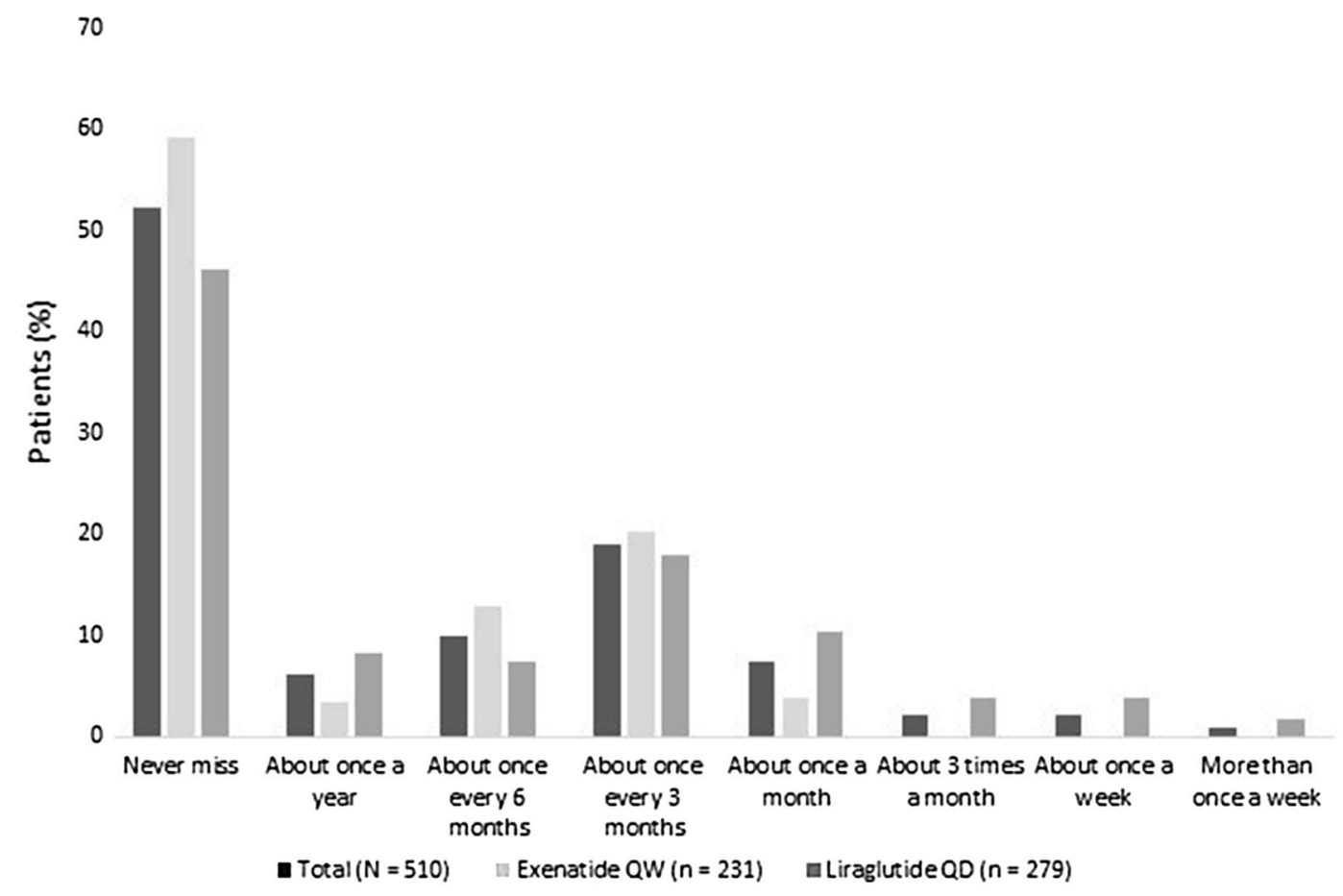

Fig. 2 Frequency of missing an injection of GLP-1 RA in injection-experienced patients with type 2 diabetes mellitus

drivers of patient preference, according to analysis of the pooled outcomes (Table 3 ).

\section{Comparison of Hypothetical GLP-1RA Profiles}

Hypothetical profiles similar to commonly prescribed GLP-1RAs with important differences in attributes were examined. Pooled data showed that when efficacy was assumed to be equal (1.2-point reduction in HbA1c) for both hypothetical treatment profiles, more injection-experienced patients preferred a GLP-1RA profile approximating exenatide $\mathrm{QW}$ administered via a single-use pen over a profile approximating liraglutide QD (Table 4). When examined by country, preference for exenatide QW (single-use pen) was higher among the German respondents than the respondents from the UK (Table 4). Even when the efficacy was assumed to be better for liraglutide QD (1.2-point reduction in HbA1c) than for single-use pen exenatide QW (0.8-point reduction in $\mathrm{HbA1C}$ ), more respondents preferred the profile approximating exenatide QW (single-use pen) than the liraglutide profile, according to the pooled data from both countries $(31.8 \%$ vs. $68.2 \%$; OR $2.15 ; p<0.001)$, the UK respondents only (38.5\% vs. $61.5 \%$; OR $1.60 ; p<0.001)$, and the German respondents only (25.6\% vs. $74.4 \%$; OR $2.91 ; p<0.001)$. 


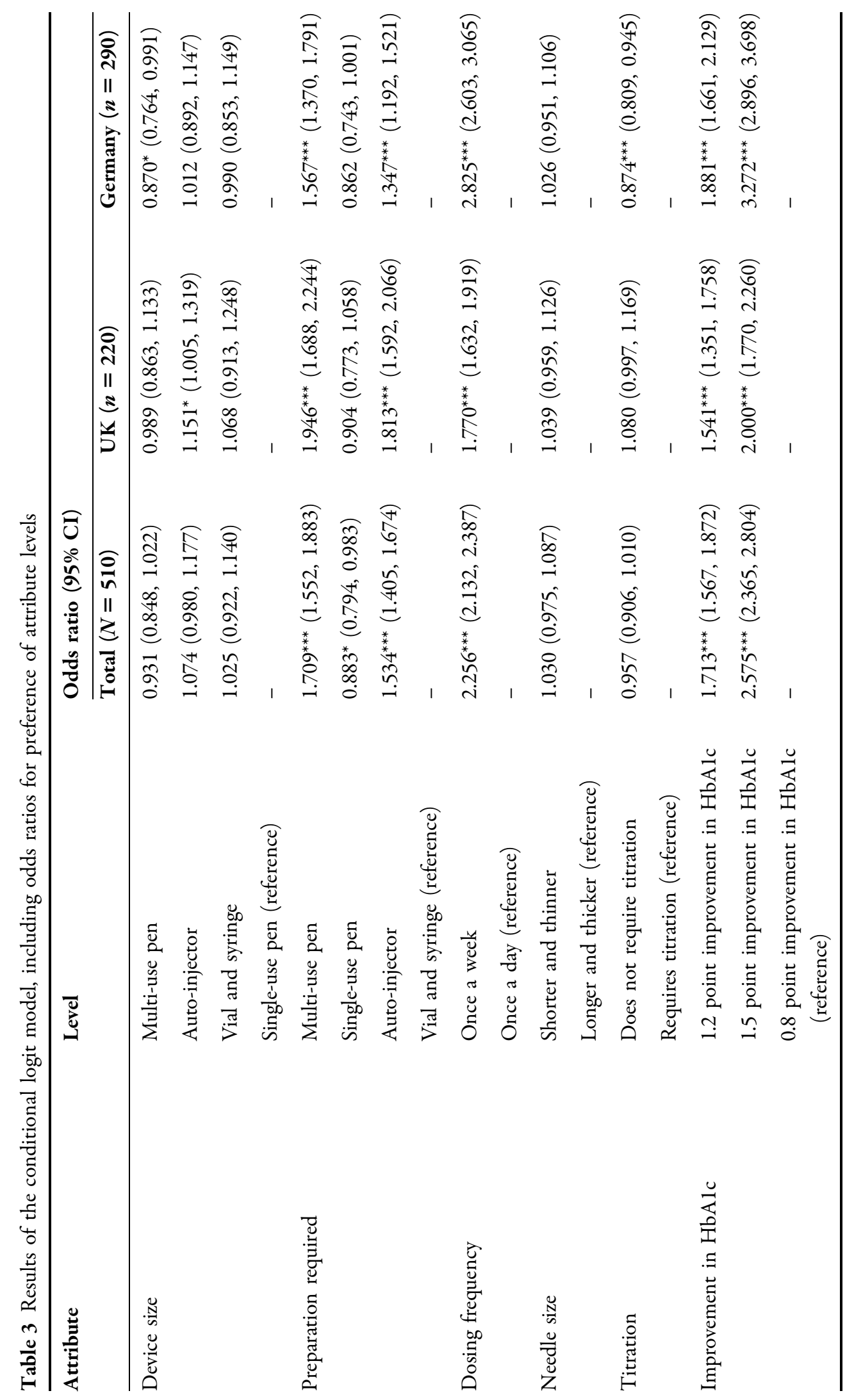




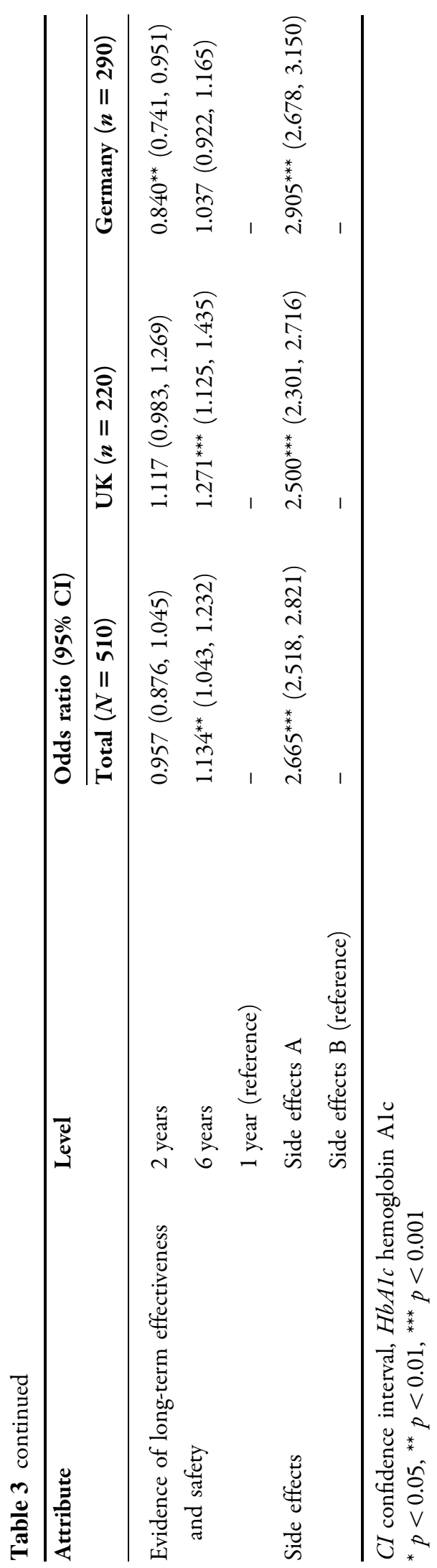

Changing device type from a single-use pen to an auto-injector pen, and assuming equal efficacy between the hypothetical profiles, increased the strength of preference for the profile approximating exenatide QW compared with a profile approximating liraglutide QD, according to an analysis across both countries (Table 5). When examined by country, preference for exenatide QW (auto-injector) was higher among the German respondents than the UK respondents (Table 5). When efficacy was assumed to be better for liraglutide QD (1.2-point improvement in $\mathrm{HbA1c}$ ) than for exenatide QW (0.8-point improvement in HbA1C), pooled results continued to show a higher preference for the profile approximating exenatide QW (auto-injector) over the profile approximating liraglutide QD (OR 4.00, $p<0.001)$. When examined by country, the preference was higher among German respondents (OR 4.59; $p<0.001)$ than among the UK respondents (OR 3.68; $p<0.001)$.

\section{DISCUSSION}

This DCE study, based on hypothetical treatment profiles, assessed the relative importance of treatment-related attributes in influencing preferences for GLP-1RAs among GLP-1RA injection-experienced patients with T2DM from Germany and the UK. Hypothetical treatment profiles were constructed based on attributes from two GLP-1RAs with important differences in attributes (i.e., exenatide QW and liraglutide QD) that were commonly used in these two countries. Across both countries, patients identified the top four most important attributes as being efficacy, side effects, dosing frequency, and required preparation. When device-related attributes were specifically examined, dosing frequency and the preparation required were significant predictors of patient preference. Needle size, device size, and titration were not significant drivers of preference for injection-experienced patients.

Other DCE studies have also examined patients' preferences for efficacy, side effects, and device-related attributes of GLP-1RAs in injection-naïve patients from the UK [31] and 


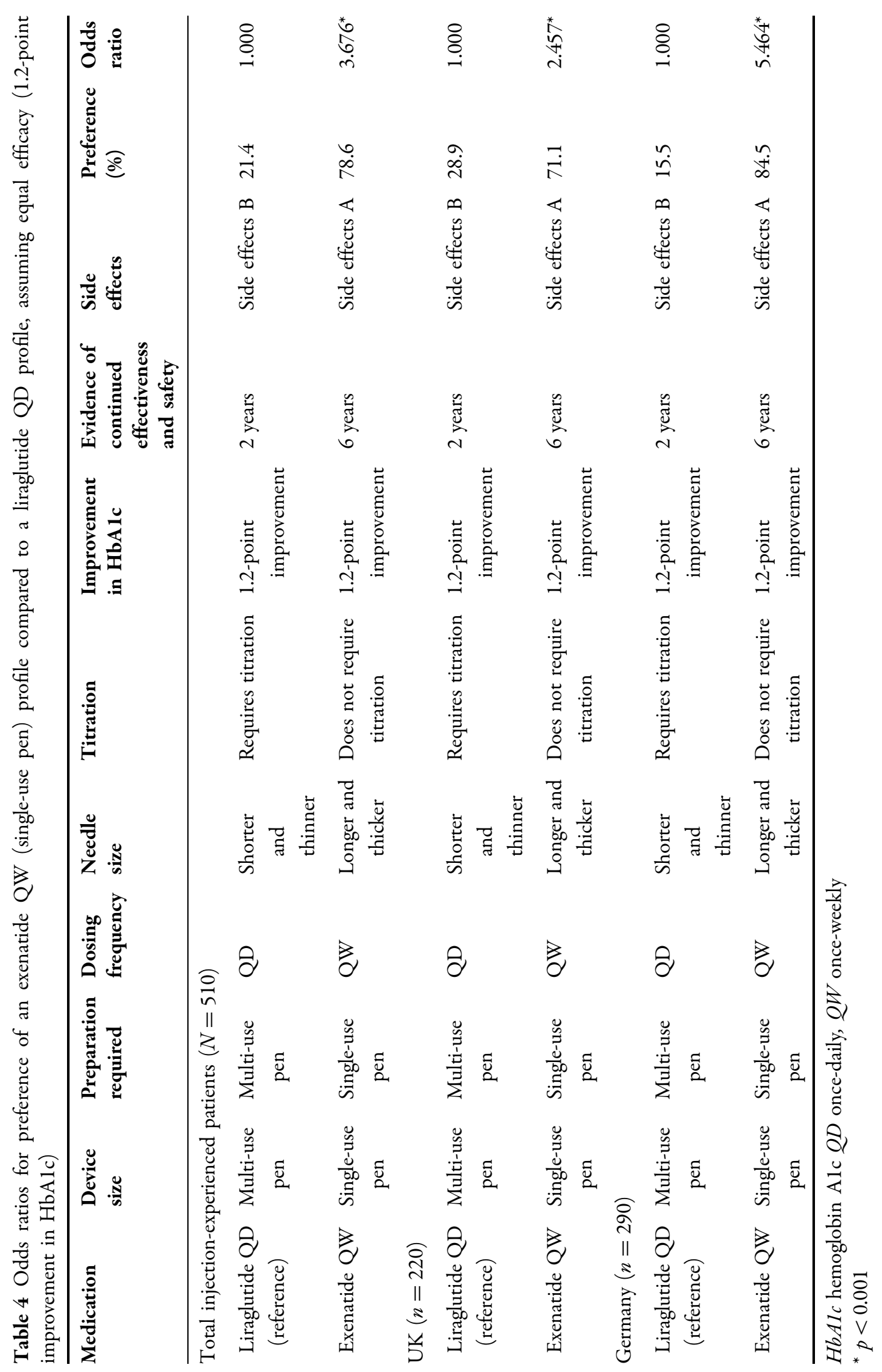




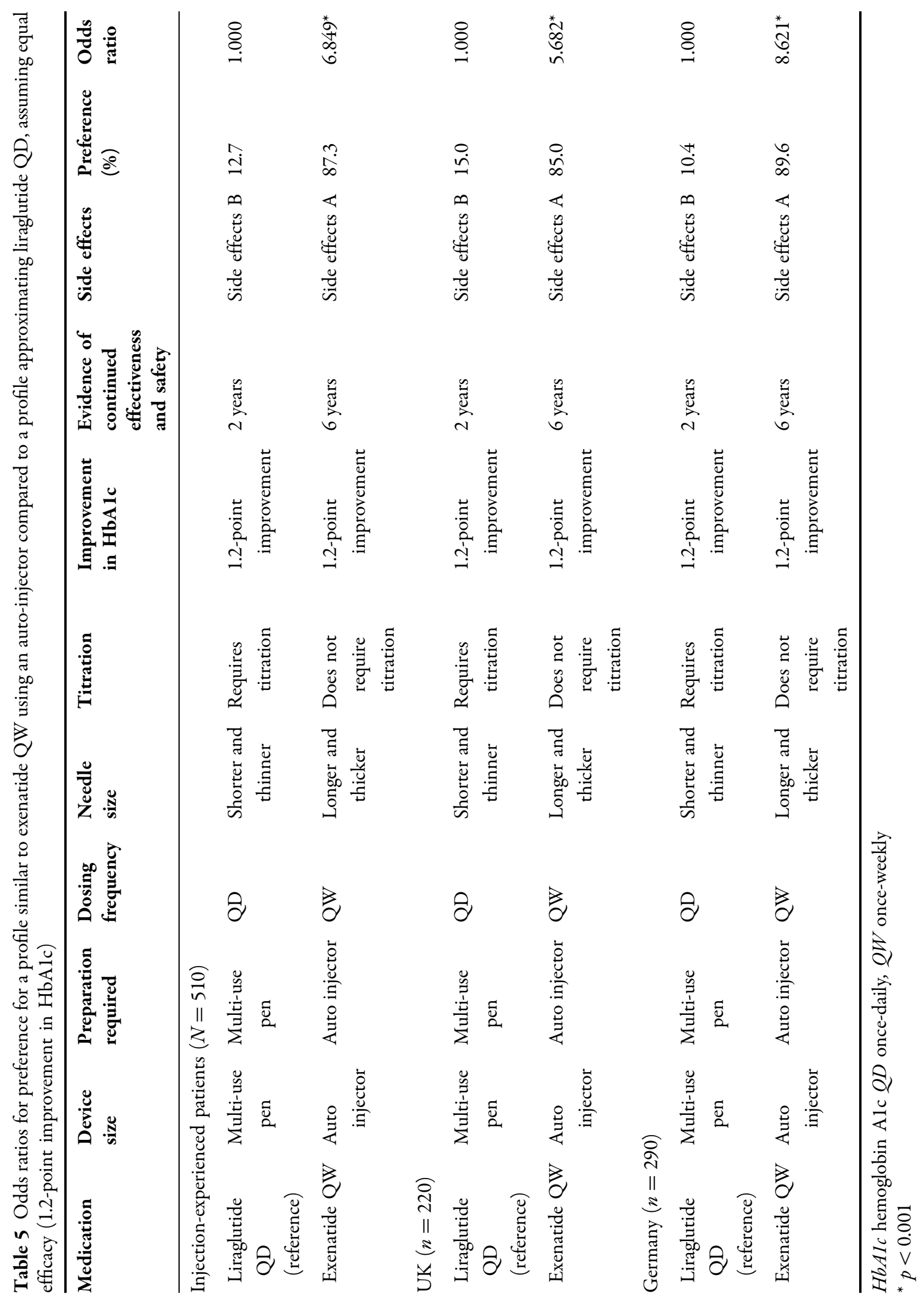


Japan [30]. Another US study in injection-experienced and injection-naïve T2DM patients focused on preferences for different dosing and device-related attributes of GLP-1RAs [33]. However, comparison of the current study with other GLP-1RA preference studies [30, 31, 33] is problematic given that many of the attributes (e.g., side effects and efficacy) were defined differently across studies. Nevertheless, outcomes from these studies provide an important context for assessing the influence of injected GLP-1RA attributes on patients' preferences. As with the current study, these studies indicated that while efficacy and tolerability attributes were important influencers of patient preference, other dosing and device-related attributes were also important [30, 31, 33].

This study in injection-experienced patients, along with a study with a similar design in injection-naïve patients [36], sought to determine if other attributes of medication (e.g., device size, the preparation required to deliver the medication, the needle size, the requirement for titration, and the evidence for longterm efficacy and safety) were also influential in determining patients' preferences. The outcomes from both of these studies were similar, with both studies indicating that side effects, efficacy, and dosing frequency were among the most important treatment attributes while needle size and device size were among the least important predictors of patient preference. However, preparation required was among the least important attributes for the injection-naïve sample, while it was an important driver of preference in the injection-experienced sample. The opposite was true for titration, which was a significant predictor of choice among the injection-naïve patients but was not so for injection-experienced patients.

Dosing frequency was an important driver of patient preference across all studies, including the current study [30, 31, 33]. Dosing frequency was the most influential attribute of GLP-1RA medication in the DCE study conducted in the US that only focused on device-related attributes in injection-experienced and injection-naïve patients [33]. The DCE studies conducted in Japan and the UK in injection-naïve patients also found that dosing frequency was among the top attributes influencing patient preference for GLP-1RAs [30, 31]. In all the GLP-1RA studies, a once-weekly dosing regimen was consistently preferred over a once-daily dosing regimen $[30,31,36,37,46$, 47]. Interestingly, DCE studies in patients with another chronic disease (multiple sclerosis; MS) also found that the frequency of injections was an important driver of preference for injectable MS treatments [48, 49].

Preparation of the GLP-1RA medication was an important aspect influencing patient preference according to outcomes from the current study. Similarly, a DCE study in patients with multiple sclerosis (MS) found that ease of use of an injection device (no need for assembly or drug reconstitution) was an important driver of treatment preferences [50].

Efficacy is ranked highly among T2DM patient preferences; however, it may not be the most important influencer of patient preference when deciding between medications that have very similar efficacies. Various studies have demonstrated equivalent efficacies of GLP-1RAs. For example, in a real-world study, changes in HbA1c were similar for those receiving exenatide $\mathrm{QW}$ and those receiving liraglutide QD over a 12-month period [51]. Similarly, a systematic review of 22 studies indicated that there was no difference between the two GLP-1RAs with regard to the level of control of HbA1c levels [52]. In this study, when efficacy was assumed to be equal between two hypothetical profiles, patients from both countries favored a GLP-1RA profile approximating exenatide QW (delivered via either a single-use pen or auto-injector) over a GLP-1RA profile approximating liraglutide QD. Similarly, injection-naïve T2DM patients from both countries indicated a strong preference for a GLP-1RA profile approximating exenatide QW (delivered via either a single-use pen or auto-injector) over a profile approximating liraglutide QD [36]. Even when the efficacies of the GLP-1RAs are not equal, patient preference for other attributes of the medication may be an important consideration. As demonstrated in this study of injection-experienced patients and in the study of injection-naïve patients [36], when the profile approximating liraglutide QD was assumed 
to have better efficacy (1.2-point vs. 0.8-point improvement in $\mathrm{HbA1c}$, respectively), patients still favored the profile approximating exenatide QW. Thus, these results indicate that attributes other than efficacy influenced patient preference for a hypothetical GLP-1RA profile.

Improvements in adherence are more likely to occur if patients' preferences for the attributes of T2DM medication are considered when clinical decisions are being made $[9,10,27]$. If patients' adherence to medication for T2DM can be improved, then improvements in diabetes-related outcomes are likely to occur, including improved glycemic control, reduced diabetes-related complications, and decreased healthcare resource utilization $[20,25,26$, 53-55].

Notably, once-weekly injections of a GLP-1RA have been associated with improved adherence compared with once-daily injections of a GLP-1RA [46, 56]. Outcomes from this global study confirmed these findings, with fewer of the patients treated with exenatide QW than those treated with liraglutide QD reporting missing doses of their medication. Potentially, omitting a single dose of a once-weekly injectable medication such as exenatide may not have a such a significant impact on overall blood glucose, given that a once-weekly injection of exenatide provides a constant 24-h exposure to the drug that is associated with improvement in HbA1c, fasting blood glucose, and postprandial hyperglycemia [57-59]. Moreover, after treatment cessation, plasma exenatide levels do not decrease to below those considered to have a therapeutic effect until after about 6 weeks. In contrast, continuous injections of liraglutide $\mathrm{QD}$ are required to maintain a constant 24-h exposure to the drug that result in the control of blood glucose levels [60]. Thus, missing a daily injection may have a more pronounced effect on blood glucose levels and overall glycemic control. However, these suppositions are yet to be confirmed in clinical studies.

A patient-centered approach that takes into account patients' preferences is needed not only during the clinical decisions that involve patients with T2DM [8-10] but also during the development of any diabetes medication, including GLP1-RAs. During the development process, injectable GLP-1RAs have improved from twice-daily injections (e.g., exenatide) to once-daily injections (e.g., liraglutide, lixisenatide), and now to once-weekly formulations (e.g., exenatide, albiglutide, and dulaglutide).

Other patient-centered improvements in injectable medication involve the development of pen devices, allowing quicker, easier, and more convenient delivery. In this study, it was not surprising that hypothetical GLP-1RA profiles including a vial and syringe were the least favored, while hypothetical profiles involving pens were more highly favored.

There were some limitations in the design of this study. DCEs may not accurately reflect real-world decisions. Due to the recruitment methods utilized for this study, the participants from Germany and the UK may not be representative of patients with T2DM in each target country. In addition, as this study was specific to Germany and the UK, the participants in this sample were predominantly Caucasian (93.5\%). There might be cultural or pathophysiological differences among racial or ethnic groups, such as Asians, that could influence preference for treatment. The results of this study might not be generalizable to other racial or ethnic groups. Liraglutide QD and exenatide QW were selected for comparison in this study because liraglutide QD is a common T2DM treatment that is used on a once-daily basis and exenatide QW, as well as being the first once-weekly T2DM treatment to be placed on the market, is commonly used in both countries. In addition, they reflect key differences in GLP1-RA treatments with different side effects and regimens. The attributes from these two specific GLP-1RA treatments were included in this survey. However, the outcomes from this study may not be generalizable to other GPL-1RA treatments with different attributes which may be available in other countries. Attributes included in the survey were limited to concepts that differed across GLP1-RAs and that were found to be important to patients. However, there may be other aspects of treatment that could influence preference for treatment, such as out-of-pocket cost or effects on overall health-related quality of life. Thus, these results may not reflect market 
share for these treatments, as other attributes may also influence treatment choice. Lastly, this study solely assessed attribute importance and treatment preferences by country. Examination of the influence of other sample characteristics, such as age and treatment experience (exenatide QW, liraglutide QD, insulin, etc.), are areas for future research.

\section{CONCLUSIONS}

This study found that the most influential drivers of treatment preference for GLP-1RAs were efficacy, side effects, dosing frequency, and required preparation among GLP-1RA injection-experienced T2DM patients in Germany and the UK. Preference elicitation can promote patient-centered care as well as inform new generations of T2DM treatments, which can lead to improved patient medication acceptance, adherence, and patient health outcomes.

\section{ACKNOWLEDGEMENTS}

Sponsorship for this study and article processing charges were funded by AstraZeneca. All named authors meet the International Committee of Medical Journal Editors (ICMJE) criteria for authorship for this manuscript, take responsibility for the integrity of the work as a whole, and have given final approval to the version to be published. All authors had full access to all of the data in this study and take complete responsibility for the integrity of the data and accuracy of the data analysis. Editorial assistance in the preparation of this manuscript was provided by Monique Curran. Support for this assistance was funded by AstraZeneca.

Disclosures. Lei Qin is employed by AstraZeneca. Stephanie Chen is employed by AstraZeneca. Susan Grandy is employed by AstraZeneca. Alka Shaunik was employed by AstraZeneca at the time of this study. Emuella Flood is employed by ICON plc, which was commissioned by AstraZeneca to perform the study. Beverly Romero is employed by ICON plc, which was commissioned by AstraZeneca to perform the study. Marie de la Cruz is employed by ICON plc, which was commissioned by AstraZeneca to perform the study. Cynthia Alvarez is employed by ICON plc, which was commissioned by AstraZeneca to perform the study.

Compliance with Ethics Guidelines. The study protocol was approved by the Salus International Review Board (Austin, TX, USA). All procedures followed were in accordance with the ethical standards of the responsible committee on human experimentation (institutional and national) and with the Helsinki Declaration of 1964, as revised in 2013. Informed consent was obtained from all patients before they were included in the study.

Data Availability. The datasets obtained during and/or analyzed during the current study are available from the corresponding author on reasonable request.

Open Access. This article is distributed under the terms of the Creative Commons Attribution-NonCommercial 4.0 International License (http://creativecommons.org/licenses/ by-nc/4.0/), which permits any noncommercial use, distribution, and reproduction in any medium, provided you give appropriate credit to the original author(s) and the source, provide a link to the Creative Commons license, and indicate if changes were made.

\section{REFERENCES}

1. NCD Risk Factor Collaboration. Worldwide trends in diabetes since 1980: a pooled analysis of 751 population-based studies with 4.4 million participants. Lancet. 2016;387:1513-30.

2. Danaei G, Finucane MM, Lu Y, et al. National, regional, and global trends in fasting plasma glucose and diabetes prevalence since 1980: systematic analysis of health examination surveys and epidemiological studies with 370 country-years and 2.7 million participants. Lancet. 2011;378: 31-40. 
3. International Diabetes Federation. IDF Diabetes Atlas, 7th edn. 2015. http://www.diabetesatlas.org. Accessed Sept 1, 2016.

4. Guariguata L, Whiting DR, Hambleton I, Beagley J, Linnenkamp U, Shaw JE. Global estimates of diabetes prevalence for 2013 and projections for 2035 . Diabetes Res Clin Pract. 2014;103:137-49.

5. Sharma M, Nazareth I, Petersen I. Trends in incidence, prevalence and prescribing in type 2 diabetes mellitus between 2000 and 2013 in primary care: a retrospective cohort study. BMJ Open. 2016;6:e010210.

6. Boehme MW, Buechele G, Frankenhauser-Mannuss $\mathrm{J}$, et al. Prevalence, incidence and concomitant co-morbidities of type 2 diabetes mellitus in South Western Germany-a retrospective cohort and case control study in claims data of a large statutory health insurance. BMC Public Health. 2015;15:855.

7. Andersson T, Ahlbom A, Magnusson C, Carlsson S. Prevalence and incidence of diabetes in Stockholm County 1990-2010. PLoS One. 2014;9:e104033.

8. Chamberlain JJ, Rhinehart AS, Shaefer CF Jr, Neuman A. Diagnosis and management of diabetes: synopsis of the 2016 American Diabetes Association Standards of Medical Care in Diabetes. Ann Intern Med. 2016;164:542-52.

9. Inzucchi SE, Bergenstal RM, Buse JB, et al. Management of hyperglycemia in type 2 diabetes, 2015: a patient-centered approach. Update to a position statement of the American Diabetes Association and the European Association for the Study of Diabetes. Diabetes Care. 2015;38:140-9.

10. Garber AJ, Abrahamson MJ, Barzilay JI, et al. Consensus statement by the American Association of Clinical Endocrinologists and American College of Endocrinology on the comprehensive type 2 diabetes management algorithm-2016 executive summary. Endocr Pract. 2016;22:84-113.

11. American Diabetes Association. Classification and diagnosis of diabetes. Diabetes Care. 2016;39(Suppl 1):S13-22.

12. American Diabetes Association. 7. Approaches to glycemic treatment. Diabetes Care. 2016;39(Suppl 1):S52-9.

13. Drucker DJ, Nauck MA. The incretin system: glucagon-like peptide-1 receptor agonists and dipeptidyl peptidase- 4 inhibitors in type 2 diabetes. Lancet. 2006;368:1696-705.

14. TrujilloJM, Nuffer W. GLP-1 receptor agonists for type 2 diabetes mellitus: recent developments and emerging agents. Pharmacotherapy. 2014;34:1174-86.
15. Trujillo JM, Nuffer W, Ellis SL. GLP-1 receptor agonists: a review of head-to-head clinical studies. Ther Adv Endocrinol Metab. 2015;6:19-28.

16. Deacon CF, Mannucci E, Ahren B. Glycaemic efficacy of glucagon-like peptide-1 receptor agonists and dipeptidyl peptidase- 4 inhibitors as add-on therapy to metformin in subjects with type 2 diabetes-a review and meta analysis. Diabetes Obes Metab. 2012;14:762-7.

17. Madsbad S. Review of head-to-head comparisons of glucagon-like peptide-1 receptor agonists. Diabetes Obes Metab. 2016;18:317-32.

18. Triplitt C, Solis-Herrera C. GLP-1 receptor agonists: practical considerations for clinical practice. Diabetes Educ. 2015;41:32s-46s.

19. Curkendall SM, Thomas N, Bell KF, Juneau PL, Weiss AJ. Predictors of medication adherence in patients with type 2 diabetes mellitus. Curr Med Res Opin. 2013;29:1275-86.

20. Garcia-Perez LE, Alvarez M, Dilla T, Gil-Guillen V, Orozco-Beltran D. Adherence to therapies in patients with type 2 diabetes. Diabetes Ther. 2013;4:175-94.

21. Grimes RT, Bennett K, Tilson L, Usher C, Smith SM, Henman MC. Initial therapy, persistence and regimen change in a cohort of newly treated type 2 diabetes patients. $\mathrm{Br} \mathrm{J}$ Clin Pharmacol. 2015;79:1000-9.

22. Kirkman MS, Rowan-Martin MT, Levin R, et al. Determinants of adherence to diabetes medications: findings from a large pharmacy claims database. Diabetes Care. 2015;38:604-9.

23. McSharry J, McGowan L, Farmer AJ, French DP. Perceptions and experiences of taking oral medications for the treatment of type 2 diabetes mellitus: a systematic review and meta-synthesis of qualitative studies. Diabet Med. 2016;33:1330-8.

24. Polonsky WH, Henry RR. Poor medication adherence in type 2 diabetes: recognizing the scope of the problem and its key contributors. Patient Prefer Adherence. 2016;10:1299-307.

25. Asche C, LaFleur J, Conner C. A review of diabetes treatment adherence and the association with clinical and economic outcomes. Clin Ther. 2011;33:74-109.

26. Doggrell SA, Warot S. The association between the measurement of adherence to anti-diabetes medicine and the HbA1c. Int J Clin Pharm. 2014;36:488-97. 
27. Ismail-Beigi F, Moghissi E, Tiktin M, Hirsch IB, Inzucchi SE, Genuth S. Individualizing glycemic targets in type 2 diabetes mellitus: implications of recent clinical trials. Ann Intern Med. 2011;154:554-9.

28. Bogelund M, Vilsboll $\mathrm{T}$, Faber J, Henriksen JE, Gjesing RP, Lammert M. Patient preferences for diabetes management among people with type 2 diabetes in Denmark-a discrete choice experiment. Curr Med Res Opin. 2011;27:2175-83.

29. Gelhorn HL, Stringer SM, Brooks A, et al. Preferences for medication attributes among patients with type 2 diabetes mellitus in the UK. Diabetes Obes Metab. 2013;15:802-9.

30. Gelhorn HL, Bacci ED, Poon JL, Boye KS, Suzuki S, Babineaux SM. Evaluating preferences for profiles of glucagon-like peptide- 1 receptor agonists among injection-naive type 2 diabetes patients in Japan. Patient Prefer Adherence. 2016;10:1337-48.

31. Gelhorn HL, Poon JL, Davies EW, Paczkowski R, Curtis SE, Boye KS. Evaluating preferences for profiles of GLP-1 receptor agonists among injection-naive type 2 diabetes patients in the UK. Patient Prefer Adherence. 2015;9:1611-22.

32. Mohamed AF, Zhang J, Johnson FR, et al. Avoidance of weight gain is important for oral type 2 diabetes treatments in Sweden and Germany: patient preferences. Diabetes Metab. 2013;39:397-403.

33. Hauber AB, Nguyen $\mathrm{H}$, Posner J, Kalsekar I, Ruggles J. A discrete-choice experiment to quantify patient preferences for frequency of glucagon-like peptide- 1 receptor agonist injections in the treatment of type 2 diabetes. Curr Med Res Opin. 2016;32:251-62.

34. Morillas C, Feliciano R, Catalina PF, et al. Patients' and physicians' preferences for type 2 diabetes mellitus treatments in Spain and Portugal: a discrete choice experiment. Patient Prefer Adherence. 2015;9:1443-58.

35. Muhlbacher A, Bethge S. What matters in type 2 diabetes mellitus oral treatment? A discrete choice experiment to evaluate patient preferences. Eur J Health Econ. 2016;17:1125-40.

36. Chen S, Qin L, Flood E, et al. Treatment attributes of GLP-1 receptor agonists important to injection-naïve patients with T2DM: a multi-national preference study. Poster 809 presented at: 52nd European Association for the Study of Diabetes Annual Meeting; 2016 Sept 12-16, Munich.

37. Bridges JF, Hauber AB, Marshall D, et al. Conjoint analysis applications in health-a checklist: a report of the ISPOR Good Research Practices for Conjoint Analysis Task Force. Value Health. 2011;14:403-13.

38. Reed Johnson F, Lancsar E, Marshall D, et al. Constructing experimental designs for discrete-choice experiments: report of the ISPOR Conjoint Analysis Experimental Design Good Research Practices Task Force. Value Health. 2013;16:3-13.

39. Grandy S, Chen S, Flood E, Romero B, Bergenheim $\mathrm{K}$, Ryden A. GLP-1 receptor agonist device- and regimen-related features important to injection-experienced and injection-naïve patients with T2DM: a multi-national interview study. ePoster \#905 presented at: 51st European Association for the Study of Diabetes Annual Meeting; 2015 Sept 15-18; Stockholm.

40. Henry RR, Klein EJ, Han J, Iqbal N. Efficacy and tolerability of exenatide once weekly over 6 years in patients with type 2 diabetes: an uncontrolled open-label extension of the DURATION-1 study. Diabetes Technol Ther. 2016;18(11):677-86.

41. Novo Nordisk A/S. Liraglutide once daily (Victoza): package insert. Bagsvaerd: Novo Nordisk A/S; 2010.

42. Amylin Pharmaceuticals Inc. Exenatide once weekly (Bydureon): package insert. San Diego: Amylin Pharmaceuticals Inc.; 2012.

43. Sloane NJA. A library of orthogonal arrays. 2016. http://neilsloane.com/oadir/. Accessed Aug 18, 2016.

44. Street DJ, Burgess L, Louviere JJ. Quick and easy choice sets: constructing optimal and nearly optimal stated choice experiments. Intern J Res Mark. 2005;22:459-70.

45. Ryan M, Bate A, Eastmond CJ, Ludbrook A. Use of discrete choice experiments to elicit preferences. Qual Health Care. 2001;10(Suppl 1):i55-60.

46. Johnston SS, Nguyen H, Felber E, et al. Retrospective study of adherence to glucagon-like peptide- 1 receptor agonist therapy in patients with type 2 diabetes mellitus in the United States. Adv Ther. 2014;31:1119-33.

47. Hauber AB, Tunceli K, Yang JC, et al. A survey of patient preferences for oral antihyperglycemic therapy in patients with type 2 diabetes mellitus. Diabetes Ther. 2015;6:75-84.

48. Poulos C, Kinter E, Yang JC, et al. A discrete-choice experiment to determine patient preferences for injectable multiple sclerosis treatments in Germany. Ther Adv Neurol Disord. 2016;9:95-104.

49. Poulos C, Kinter E, Yang JC, Bridges JF, Posner J, Reder AT. Patient preferences for 
injectable treatments for multiple sclerosis in the United States: a discrete-choice experiment. Patient. 2016;9:171-80.

50. Shingler SL, Swinburn P, Ali S, Perard R, Lloyd AJ. A discrete choice experiment to determine patient preferences for injection devices in multiple sclerosis. J Med Econ. 2013;16:1036-42.

51. Saunders WB, Nguyen H, Kalsekar I. Real-world glycemic outcomes in patients with type 2 diabetes initiating exenatide once weekly and liraglutide once daily: a retrospective cohort study. Diabetes Metab Syndr Obes. 2016;9:217-23.

52. Scott DA, Boye KS, Timlin L, Clark JF, Best JH. A network meta-analysis to compare glycaemic control in patients with type 2 diabetes treated with exenatide once weekly or liraglutide once daily in comparison with insulin glargine, exenatide twice daily or placebo. Diabetes Obes Metab. 2013;15:213-23.

53. Buysman EK, Liu F, Hammer M, Langer J. Impact of medication adherence and persistence on clinical and economic outcomes in patients with type 2 diabetes treated with liraglutide: a retrospective cohort study. Adv Ther. 2015;32:341-55.

54. Boye KS, Curtis SE, Lage MJ, Garcia-Perez LE. Associations between adherence and outcomes among older, type 2 diabetes patients: evidence from a Medicare supplemental database. Patient Prefer Adherence. 2016;10:1573-81.
55. Simpson SH, Lin M, Eurich DT. Medication adherence affects risk of new diabetes complications: a cohort study. Ann Pharmacother. 2016;50:741-6.

56. Qiao Q, Ouwens MJ, Grandy S, Johnsson K, Kostev K. Adherence to GLP-1 receptor agonist therapy administered by once-daily or once-weekly injection in patients with type 2 diabetes in Germany. Diabetes Metab Syndr Obes. 2016;9:201-5.

57. Drucker DJ, Buse JB, Taylor K, et al. Exenatide once weekly versus twice daily for the treatment of type 2 diabetes: a randomised, open-label, non-inferiority study. Lancet. 2008;372:1240-50.

58. Iwamoto K, Nasu R, Yamamura A, et al. Safety, tolerability, pharmacokinetics, and pharmacodynamics of exenatide once weekly in Japanese patients with type 2 diabetes. Endocr J. 2009;56:951-62.

59. Kim D, MacConell L, Zhuang D, et al. Effects of once-weekly dosing of a long-acting release formulation of exenatide on glucose control and body weight in subjects with type 2 diabetes. Diabetes Care. 2007;30:1487-93.

60. Elbrond B, Jakobsen G, Larsen S, et al. Pharmacokinetics, pharmacodynamics, safety, and tolerability of a single-dose of NN2211, a long-acting glucagon-like peptide 1 derivative, in healthy male subjects. Diabetes Care. 2002;25:1398-404. 